\title{
Nanoparticle albumin-bound paclitaxel and ramucirumab versus paclitaxel and ramucirumab as second-line chemotherapy for unresectable advanced or recurrent gastric cancer: a multicenter, propensity score-matched analysis (CROSS SELL study)
}

\author{
Akio Nakasya ${ }^{1}$ (1) $\cdot$ Yuya Hagiwara ${ }^{2} \cdot$ Tatsuki lkoma $^{3} \cdot$ Yusuke Kurioka $^{4} \cdot$ Toshihiko Matsumoto $^{3,4}$. \\ Yoshiyuki Yamamoto ${ }^{2}$. Takao Tsuduki ${ }^{4} \cdot$ Takeshi Kajiwara $^{1} \cdot$ Toshikazu Moriwaki $^{2} \cdot$ Tomohiro Nishina $^{1}$. \\ Natsumi Yamashita ${ }^{5}$. Ichinosuke Hyodo ${ }^{1}$
}

Received: 15 October 2021 / Accepted: 3 January 2022 / Published online: 28 January 2022

(c) The Author(s) 2022

\begin{abstract}
Background Paclitaxel plus ramucirumab (PTX + RAM) is the standard second-line chemotherapy for unresectable advanced or recurrent gastric cancer (AGC). Nanoparticle albumin-bound paclitaxel (nab-PTX) is an improved, more convenient form of PTX and is non-inferior to PTX. Although some retrospective and single-arm phase II studies regarding nab-PTX + RAM have been reported, comparative studies are lacking. Here, we compared the efficacy and toxicity of nab-PTX + RAM and PTX + RAM using propensity score matching.

Methods Clinical data of 265 patients treated for AGC with nab-PTX + RAM or PTX + RAM were retrospectively collected. Nab-PTX was administered at dosages of $100 \mathrm{mg} / \mathrm{m}^{2}$, replacing PTX in the standard PTX + RAM regimen. Progression-free survival (PFS), overall survival (OS), and toxicity were compared using 1:1 propensity score matching.

Results In total, 190 (72\%) patients were matched. The median PFS was 5.3 [95\% confidence interval (CI) 4.4-6.3] and 4.7 (95\% CI 3.2-5.3) months in the nab-PTX + RAM and PTX + RAM groups, respectively [hazard ratio $(\mathrm{HR})=0.76,95 \% \mathrm{CI}$ $0.56-1.03, p=0.07]$. The median OS was 11.5 (95\% CI 9.2-15.0) and 9.9 (95\% CI 8.0-12.7) months, respectively (HR=0.78, $95 \%$ CI $0.56-1.07, p=0.12$ ). Grade 3 and 4 neutropenia was observed more frequently in the nab-PTX + RAM group (72\% vs. $56 \%, p=0.03)$. No treatment-related deaths occurred.

Conclusions Nab-PTX + RAM exhibited more favorable trends in terms of PFS and OS but was more myelosuppressive than PTX + RAM. As neutropenia is commonly manageable toxicity, nab-PTX + RAM presents a treatment alternative for AGC. Further studies including randomized, controlled studies are warranted.
\end{abstract}

Keywords Gastric cancer · Chemotherapy · Albumin-bound paclitaxel · Paclitaxel · Ramucirumab · Propensity score

Akio Nakasya

nakashiya.akio.nt@mail.hosp.go.jp

1 Department of Gastrointestinal Medical Oncology, National Hospital Organization Shikoku Cancer Center, 160 Kou, Minamiumemoto-machi, Matsuyama, Ehime 791-0280, Japan

2 Department of Gastroenterology, Faculty of Medicine, University of Tsukuba, Tsukuba, Ibaraki, Japan
3 Department of Medical Oncology, Kobe City Medical Center General Hospital, Kobe, Hyogo, Japan

4 Department of Internal Medicine, Himeji Red Cross Hospital, Himeji, Hyogo, Japan

5 Section of Cancer Prevention and Epidemiology, Clinical Research Center, National Hospital Organization Shikoku Cancer Center, Matsuyama, Ehime, Japan 


\section{Introduction}

Fluoropyrimidine plus platinum is the recommended standard first-line chemotherapy for unresectable advanced or recurrent gastric cancer (AGC) according to several guidelines [1-3]. The RAINBOW trial [4] demonstrated the superiority of weekly administration of paclitaxel plus ramucirumab (PTX + RAM) over weekly PTX in overall survival (OS) in previously treated AGC patients, after which PTX + RAM became the standard second-line chemotherapy.

Nanoparticle albumin-bound paclitaxel (nab-PTX) is a solvent-free albumin-bound, $130 \mathrm{~nm}$ particle form of PTX. Since nab-PTX is free of polyethoxylated castor oil and hydrated ethanol, patients are at lower risk of hypersensitivity compared to when PTX is administered [5]. Therefore, nab-PTX is administered for a shorter time than PTX without premedication and patients with alcohol intolerance can be treated with it [5]. Thus, nab-PTX is more beneficial and convenient than PTX in clinical practice.

The ABSOLUTE trial demonstrated the non-inferiority of weekly nab-PTX to weekly PTX in OS (median, 11.1 vs. 10.9 months; hazard ratio $[\mathrm{HR}=0.97,97.5 \%$ confidence interval (CI) 0.76-1.23; non-inferiority $p=0.0085$ ], and improved trends in progression-free survival (PFS) (respective median, 5.3 vs. 3.8 months; $\mathrm{HR}=0.88,95 \% \mathrm{CI}$ $0.73-1.06 ; p=0.17)$ and overall response rate (ORR) $(33 \%$ vs. $24 \%, p=0.10$ ) as second-line chemotherapy for AGC [5]. In addition, a single-arm phase II trial of nab-PTX + RAM and two retrospective studies of nab-PTX + RAM and PTX + RAM demonstrated promising and similar efficacy, respectively, as second-line chemotherapy for AGC [6-8]. Thus, nab-PTX + RAM is expected to be an alternative treatment to PTX-RAM and could be used instead of PTX + RAM in Japan. However, no comparative study of nab-PTX-RAM and PTX-RAM has been conducted thus far.

Here, we retrospectively analyzed the outcomes of the two treatments using propensity score matching to minimize the bias of patient backgrounds.

\section{Patients and methods}

\section{Study design and patients}

This was a multicenter retrospective study conducted at four institutions (National Hospital Organization, Shikoku Cancer Center, Matsuyama, Ehime, Japan; University of Tsukuba, Tsukuba, Ibaraki, Japan; Himeji Red Cross Hospital, Himeji, Hyogo, Japan; Kobe City Medical Center General Hospital, Kobe, Hyogo, Japan).
The major inclusion criteria were as follows: (a) unresectable advanced or recurrent gastric cancer (including esophagogastric junction cancer), (b) histologically confirmed adenocarcinoma, (c) age: $\geq 20$ years, (d) Eastern Cooperative Oncology Group performance status (ECOG PS) of 0-2, (e) evaluable lesions, (f) refractoriness to firstline chemotherapy with the fluoropyrimidine-based regimen (including relapse $\leq 24$ weeks after the final administration of fluoropyrimidine-based adjuvant chemotherapy), (g) receiving nab-PTX + RAM or PTX + RAM as second-line chemotherapy, and (h) initiation of second-line chemotherapy between January 2017 and June 2020. The major exclusion criteria were as follows: (i) history of previous administration of taxane or angiogenesis inhibitors and (j) lost to follow-up within 1 month of starting nab-PTX + RAM or PTX + RAM treatment.

\section{Treatment}

The nab-PTX + RAM regimen consisted of administration of $100 \mathrm{mg} / \mathrm{m}^{2}$ of nab-PTX intravenously over $30 \mathrm{~min}$ on days 1,8 , and 15 along with $8 \mathrm{mg} / \mathrm{kg}$ of RAM intravenously on days 1 and 15 of each 28-day cycle. The only premedication permitted was the histamine H1-receptor blocker prior to RAM infusion on days 1 and 15. The PTX + RAM regimen consisted of administration of $80 \mathrm{mg} / \mathrm{m}^{2}$ of PTX intravenously over 60 min on days 1,8 , and 15 , along with $8 \mathrm{mg} / \mathrm{kg}$ of RAM intravenously on days 1 and 15 of each 28-day cycle. The permitted premedication was steroids and histamine $\mathrm{H} 1$ and $\mathrm{H} 2$-receptor blockers on days 1, 8, and 15. The attending physician determined each patient's regimen. Nab-PTX + RAM was preferentially selected for the patients with alcohol intolerance, allergies, and underlying conditions (diabetes mellitus, non-tuberculosis mycobacterial infection, and so on) to avoid steroid use. In addition, drug cost, physician's experience, and institution policy to reduce treatment time affected the choice of regimen. Dose reductions, including the initial dose, and skipping or delaying administration, were also determined according to each physician's discretion. Treatment was continued until disease progression, unacceptable toxicity, patient refusal, or a physician's decision to discontinue.

\section{Endpoints and assessment}

Efficacy was evaluated based on PFS, OS, and tumor response. Toxicity was evaluated according to the proportion of patients with Grade 3 or 4 adverse events (AEs). PFS was defined as the time from the initiation of study treatment to disease progression or death due to any cause. Disease progression was defined as radiological or clinical cancer progression. The patients underwent radiological examination 
every $8 \pm 2$ weeks. Patients who continued study treatment and discontinued treatment without disease progression were censored at the last confirmation of non-progressive disease by radiological examination. OS was defined as the time from the initiation of study treatment to death due to any cause. Survivors were censored at last contact. Tumor response was assessed based on the Response Evaluation Criteria in Solid Tumors version 1.1 (RECIST ver. 1.1) [9] for patients with measurable lesions. ORR was defined as the proportion of patients who had the best response of complete response or partial response. Disease control rate (DCR) was defined as the proportion of patients who had the best response of complete response, partial response, or stable disease. AEs were graded based on the Common Terminology Criteria for Adverse Events version 5.0 (CTCAE ver. 5.0) [10]. Disease progression was decided by each physician. Tumor response and AEs were also assessed by each physician. Relative dose intensity (RDI) was defined as the ratio of actually delivered dose to the standard dose of drugs from the first to the last administration.

\section{Statistical analysis}

At first, we defined all patients who met the inclusion and exclusion criteria as the original cohort. Patient backgrounds and treatments are both known to affect efficacy and survival $[11,12]$. Therefore, we used 1:1 propensity score matching to balance the patient background characteristics between the two treatment groups. Propensity scores were estimated using a multivariable logistic regression model that included six covariates [ECOG PS, histological tumor differentiation, presence of primary tumor, number of metastatic sites, peritoneal metastasis, and serum lactate dehydrogenase (LDH) level]. These covariates were identified by the multivariate analysis for OS in the original cohort (cutoff $p<0.20$ ), and adopted confirming correspondence to the reported prognostic factors [11-13]. Then, the patients matched using the scores were defined as the matched cohort. The patient characteristics of the two treatment groups were compared using standardized differences. The efficacy and toxicity of the groups were compared in the matched cohort. Inverse probability of treatment weighting (IPTW) analysis was also performed as sensitivity analysis in the original cohort. Survival curves were generated using the Kaplan-Meier method. The PFS and OS rates were compared using the log-rank test. HR and 95\% CI were estimated using the Cox proportional hazards model, as was subgroup univariate analysis. Fisher's exact test was used to compare the ORR, DCR and toxicity of the groups. Wilcoxon rank sum test was used to compare the RDI and actually delivered dose of the groups. The follow-up time was estimated using the reverse Kaplan-Meier method. A standardized difference of $<0.10$ was defined as statistically not different or well balanced
[14]. All $p$ values were two-sided, and statistical significance was set at $p<0.05$. Statistical analyses were performed using JMP $^{\circledR} 13$ (SAS Institute Inc., Cary, NC, USA) and SAS software (version 9.4; SAS Institute Inc., Cary, NC, USA).

\section{Results}

\section{Patients}

The flow chart for patient selection is shown in Fig. 1. Data of 265 patients treated with nab-PTX + RAM or PTX + RAM as second-line chemotherapy were collected from four institutions. After ineligible patients were excluded, 129 and 126 patients from the nab-PTX + RAM and PTX + RAM groups, respectively, were analyzed as the original cohort. The background characteristics of the patients in each treatment group are shown in Table 1. There were some imbalances between the two treatment groups, with the nab-PTX + RAM group exhibiting worse background characteristics (undifferentiated tumor, multiple number of metastatic sites, presence of primary tumor, peritoneal metastasis, and a high LDH level). After 1:1 propensity score matching, 190 (72\%) patients were matched as 95 pairs. In this matched cohort, the patient background characteristics were balanced between the two treatment groups (Table 1).

\section{Efficacy}

In the matched cohort, 94 (99\%) and 95 (100\%) patients in the nab-PTX-RAM and PTX + RAM groups, respectively, discontinued study treatment. The most common reason for

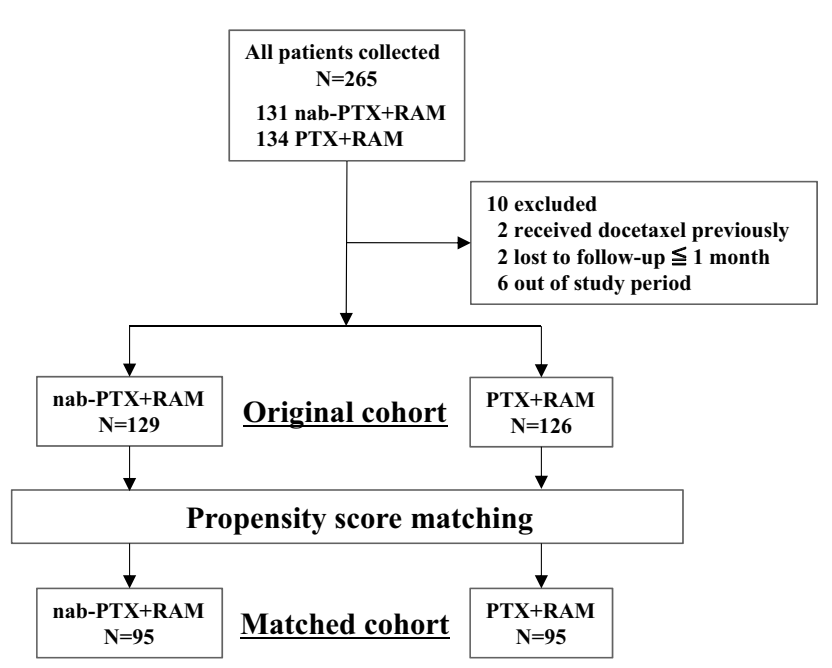

Fig. 1 Consolidated Standards of Reporting Trial (CONSORT) flow diagram. Nab-PTX+RAM nanoparticle albumin-bound paclitaxel plus ramucirumab, $P T X+R A M$ paclitaxel plus ramucirumab, $N$ number of patients 
Table 1 Patient background characteristics

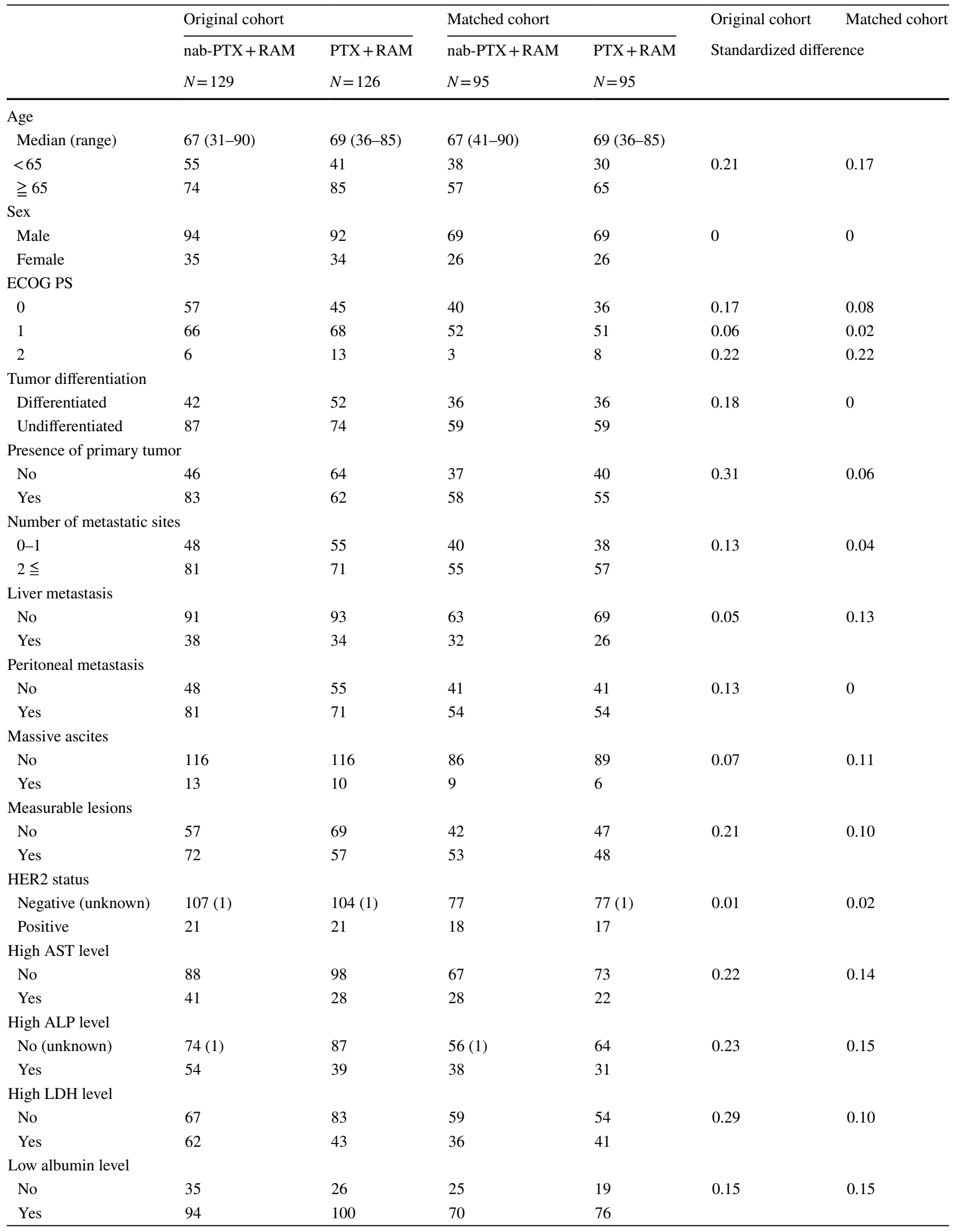


Table 1 (continued)

\begin{tabular}{|c|c|c|c|c|c|c|}
\hline & \multicolumn{2}{|l|}{ Original cohort } & \multicolumn{2}{|l|}{ Matched cohort } & \multirow{3}{*}{$\begin{array}{l}\text { Original cohort } \\
\text { Standardized dif }\end{array}$} & \multirow{3}{*}{$\begin{array}{l}\text { Matched cohort } \\
\text { nce }\end{array}$} \\
\hline & nab-PTX + RAM & PTX + RAM & nab-PTX + RAM & PTX + RAM & & \\
\hline & $N=129$ & $N=126$ & $N=95$ & $N=95$ & & \\
\hline \multicolumn{7}{|l|}{ Low sodium level } \\
\hline No & 117 & 114 & 86 & 88 & 0.01 & 0.07 \\
\hline Yes & 12 & 12 & 9 & 7 & & \\
\hline \multicolumn{7}{|l|}{ High neutrophil level } \\
\hline No (unknown) & 100 & $98(1)$ & 74 & $75(1)$ & 0.03 & 0.05 \\
\hline Yes & 29 & 27 & 21 & 19 & & \\
\hline \multicolumn{7}{|c|}{ Low lymphocyte level } \\
\hline No (unknown) & 105 & $98(1)$ & 79 & $73(1)$ & 0.07 & 0.13 \\
\hline Yes & 24 & 27 & 16 & 21 & & \\
\hline \multicolumn{7}{|c|}{ First-line chemotherapy } \\
\hline Fluoropyrimidine & 129 & 126 & 95 & 95 & 0 & 0 \\
\hline Platinum & 115 & 98 & 84 & 75 & 0.31 & 0.25 \\
\hline
\end{tabular}

$N a b-P T X+R A M$ nanoparticle albumin-bound paclitaxel plus ramucirumab, $P T X+R A M$ paclitaxel plus ramucirumab, $N$ number of patients, ECOG PS Eastern Cooperative Oncology Group performance status, HER2 human epidermal growth factor receptor 2, AST aspartate aminotransferase, $A L P$ alkaline phosphatase, $L D H$ lactate dehydrogenase, massive ascites means that ascites exist from the surface of the liver to the pelvic cavity continuously

treatment discontinuation was disease progression, which affected $88(93 \%)$ patients in each group (Online Resource 1). At the data cutoff time for analyses (September 2021), the median follow-up time for OS was 24.8 and 29.9 months in the nab-PTX + RAM and PTX + RAM groups, respectively. During the study period, $72(76 \%)$ and $82(86 \%)$ patients died in the nab-PTX + RAM and PTX + RAM groups, respectively.

The median PFS was 5.3 (95\% CI 4.4-6.3) and 4.7 (95\% CI 3.2-5.3) months in the nab-PTX + RAM and PTX + RAM groups, respectively $(\mathrm{HR}=0.76,95 \% \mathrm{CI}$ $0.56-1.03, p=0.07$ ) (Fig. 2a). The median OS was 11.5 (95\% CI: 9.2-15.0) months in the nab-PTX + RAM group and 9.9 (95\% CI 8.0-12.7) months in the PTX + RAM group (HR $=0.78,95 \%$ CI 0.56-1.07, $p=0.12$ ) (Fig. 2b). According to IPTW analysis, the HR of nab-PTX + RAM versus PTX + RAM for PFS was 0.86 (95\% CI 0.65-1.13, $p=0.28)$ and that for OS was 0.83 (95\% CI 0.62-1.11, $p=0.21$ ) (Fig. 3). Subgroup analyses generally tended to favor nab-PTX + RAM in terms of PFS and OS (Fig. 4). The ORR was $40 \%$ and $37 \%$ in the nab-PTX + RAM and PTX + RAM groups, respectively $(p=0.84)$, and the DCR was $87 \%$ and $77 \%(p=0.29)$, respectively (Table 2$)$. Online Resource 2 shows the waterfall plots of tumor shrinkage of each patient. The mean depth of response was $-21 \%$ (95\% CI $-12 \%$ to $-30 \%$ ) in the nab-PTX + RAM group and $-14 \%$ (95\% CI $-3 \%$ to $-25 \%)$ in the PTX+RAM group.

\section{Toxicity}

The proportion of patients with Grade 3 or 4 AEs in the nab-PTX + RAM group was higher than in the PTX + RAM group ( $86 \%$ vs. $69 \%, p<0.01)$ (Table 3 ). The most common AE was a decrease in neutrophil count and more patients in the nab-PTX + RAM group developed this condition than in the PTX + RAM group (72\% vs. $56 \%, p=0.03)$. The incidence of febrile neutropenia was comparable between the groups ( $8 \%$ vs. $9 \%, p=1.00$ ). Grade 3 and 4 anemia tended to occur more frequently in the nab-PTX + RAM group than in the PTX + RAM group ( $26 \%$ vs. $17 \%, p=0.15$ ). No hypersensitivity reactions or treatment-related deaths occurred during either treatment.

\section{RDI and actually delivered dose}

The median RDI of taxane was $63 \%$ [interquartile range (IQR) 49-83] and 66\% (IQR 51-83) in the nabPTX + RAM and PTX + RAM groups, respectively $(p=0.50)$. The median actually delivered dose of taxane was $190 \mathrm{mg} / \mathrm{m}^{2} /$ cycle (IQR $147-249$ ) and $158 \mathrm{mg} / \mathrm{m}^{2} /$ cycle (IQR 121-199) in the nab-PTX + RAM and PTX + RAM groups, respectively $(p<0.01)$. The median RDI of RAM was $85 \%$ (IQR 67-100) and $89 \%$ (IQR 71-100) in the nab-PTX + RAM and PTX + RAM groups, respectively 
Fig. 2 Kaplan-Meier curves of progression-free survival (a) and overall survival (b) in propensity score-matched patients. $N a b-P T X+R A M$ nanoparticle albumin-bound paclitaxel plus ramucirumab, $P T X+R A M$ paclitaxel plus ramucirumab, $H R$ hazard ratio, $C I$ confidence interval

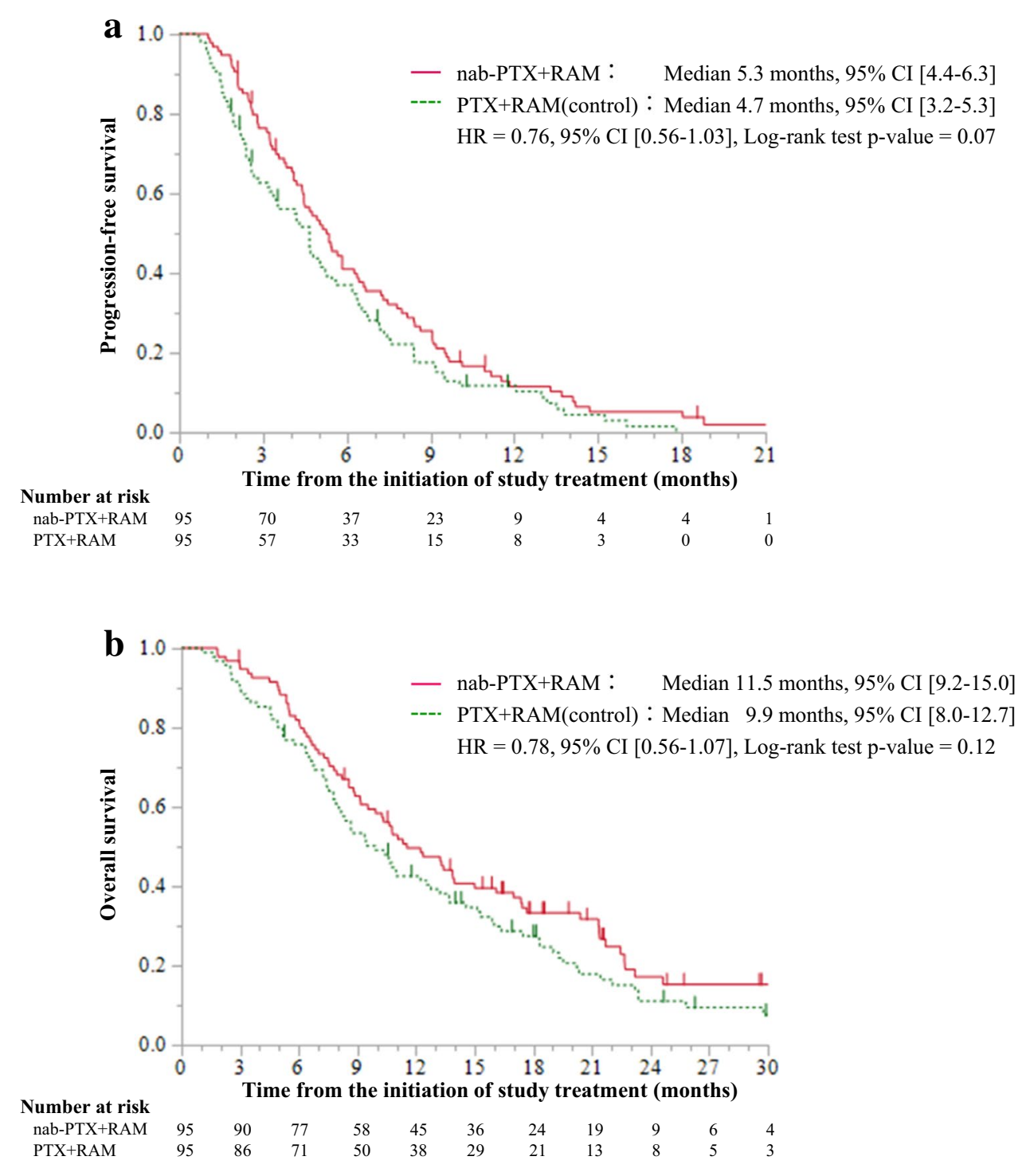

$(p=0.28)$. The median actually delivered dose of RAM was $169 \mathrm{mg} / \mathrm{m}^{2} /$ cycle (IQR: $134-200$ ) and $178 \mathrm{mg} /$ $\mathrm{m}^{2} /$ cycle (IQR: $141-200$ ) in the nab-PTX + RAM and PTX + RAM groups, respectively $(p=0.28)$.

\section{Post-study treatment}

Approximately $81 \%$ and $64 \%$ of patients in the nabPTX + RAM and PTX + RAM groups received third-line chemotherapy and $40 \%$ and $34 \%$ received fourth-line chemotherapy, respectively. The proportions of patients by treatments in the nab-PTX + RAM and PTX + RAM groups were as follows: immune checkpoint inhibitors (nivolumab or pembrolizumab), $72 \%$ and $57 \%$; irinotecan, $29 \%$ and $27 \%$; trifluridine/tipiracil, 24\% and 11\%; and trastuzumab deruxtecan, $11 \%$ and 5\% (Online Resource 3).

\section{Discussion}

To our knowledge, this is the first study to compare the efficacy and toxicity of nab-PTX + RAM versus PTX + RAM as second-line chemotherapy in multicenter and propensity score-matched patients with AGC. Nab-PTX+RAM 
exhibited more favorable trends in efficacy and more myelosuppressive AEs in toxicity, compared with PTX + RAM.

In terms of efficacy, nab-PTX + RAM exhibited favorable trends in PFS and OS compared to PTX + RAM, despite no statistically significant differences being observed. Sensitivity analyses using IPTW revealed similar results. The PFS and OS rates of the PTX + RAM group in our study were comparable to those observed in the RAINBOW trial [4]. In addition, as shown in Online Resource 4, the PFS and OS of nab-PTX + RAM in the previous two retrospective studies appeared favorable compared with those of PTX + RAM, or at least non-inferior. Forest plots of subgroup analyses of PFS and OS generally tended to favor nab-PTX + RAM. Nab-PTX appeared to have a better effect on peritoneal metastasis than PTX according to the exploratory subgroup analysis of the ABSOLUTE trial [15] and a retrospective study on nab-PTX + RAM and PTX-RAM [7], presumably due to the drug formulation of nab-PTX. However, this interaction between peritoneal metastasis and treatment was not observed in our study or in another retrospective study [8], and remained controversial. The ongoing P-SELECT trial (WJOG10617G) [16], a multicenter randomized phase II trial of nab-PTX + RAM versus PTX + RAM in second-line chemotherapy for AGC patients with peritoneal metastasis, may elucidate this finding. The current study demonstrated that ORR, DCR, and depth of response were comparable between the two treatments, which is equivalent to the results of other studies [7, 8].

Regarding toxicity, the proportion of patients with Grade 3 and 4 AEs was statistically higher in the nab-PTX + RAM group than in the PTX + RAM group. These were predominantly manageable hematological toxicities, such as a neutrophil count decrease and anemia. Although the RDIs of taxane and RAM were compatible between the two treatments, the actually delivered dose of taxane was statistically higher in the nab-PTX + RAM group than that in the PTX + RAM group, which seemed to explain the increase of hematological toxicities in the nab-PTX + RAM group. A retrospective study showed that the neutrophil count decrease observed in the patients receiving weekly PTX was strongly associated with better efficacy [17], and a prospective study demonstrated that PFS rates were better in patients treated with neutropenia-guided dose-escalation weekly PTX than in those receiving standard-dose weekly PTX [18]. Taken together with these findings, the higher incidence of neutrophil count decreased in nab-PTX + RAM might not be a disadvantage for efficacy. Considering that
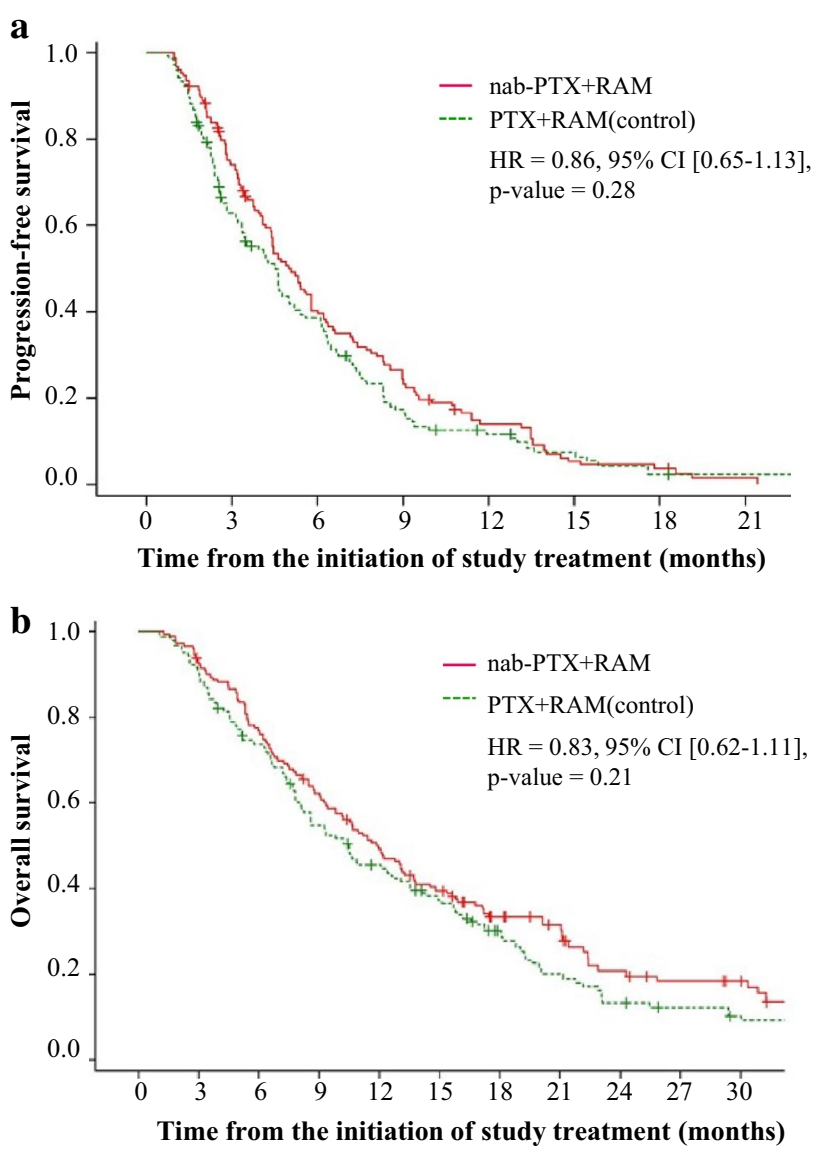

Fig. 3 Kaplan-Meier curves of progression-free survival (a) and overall survival (b) in Inverse Probability of Treatment Weighting (IPTW) patients. Nab-PTX $+R A M$ nanoparticle albumin-bound paclitaxel plus ramucirumab, $P T X+R A M$ paclitaxel plus ramucirumab, $H R$ hazard ratio, $C I$ confidence interval

less than $10 \%$ of patients discontinued study treatment due to unacceptable toxicity and that treatment-related death did not occur due to either treatment, both treatments appear feasible in clinical practice. No hypersensitivity reactions were observed in either treatment group. As indicated in "Methods", the only permitted premedication for nab-PTX + RAM was a histamine H1-receptor blocker, making this result remarkable. In addition, nab-PTX can be used in patients with alcohol intolerance and has a shorter administration time. Nab-PTX + RAM is, therefore, more convenient than PTX + RAM in clinical practice.

The proportions of patients who received later-line chemotherapy, primarily immune checkpoint inhibitors 
Fig. 4 Forest plots for subgroup univariate analyses of progression-free survival (a) and overall survival (b). The size of the center circle is proportional to the number of patients in the subgroup. Nab-PTX+RAM nanoparticle albumin-bound paclitaxel plus ramucirumab, $P T X+R A M$ paclitaxel plus ramucirumab, $H R$ hazard ratio, $C I$ confidence interval, ECOG PS Eastern Cooperative Oncology Group performance status, HER2 human epidermal growth factor receptor 2, AST aspartate aminotransferase, $A L P$ alkaline phosphatase, $L D H$ lactate dehydrogenase

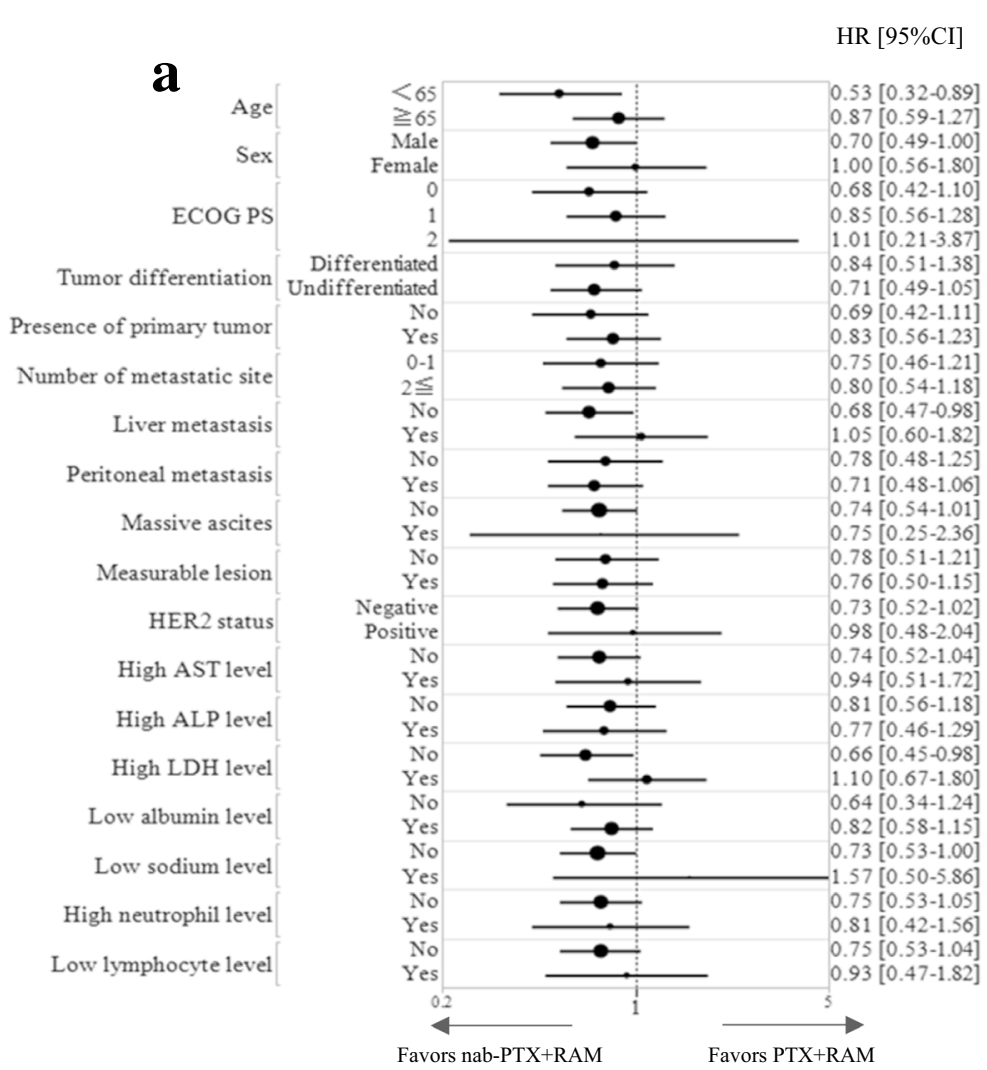

Interaction p-value

0.13

0.31

0.57

0.42

0.43

0.78

0.27

0.72

0.94

0.96

0.48

0.78

0.65

0.09

0.59

0.32

0.64

0.55

Favors PTX+RAM

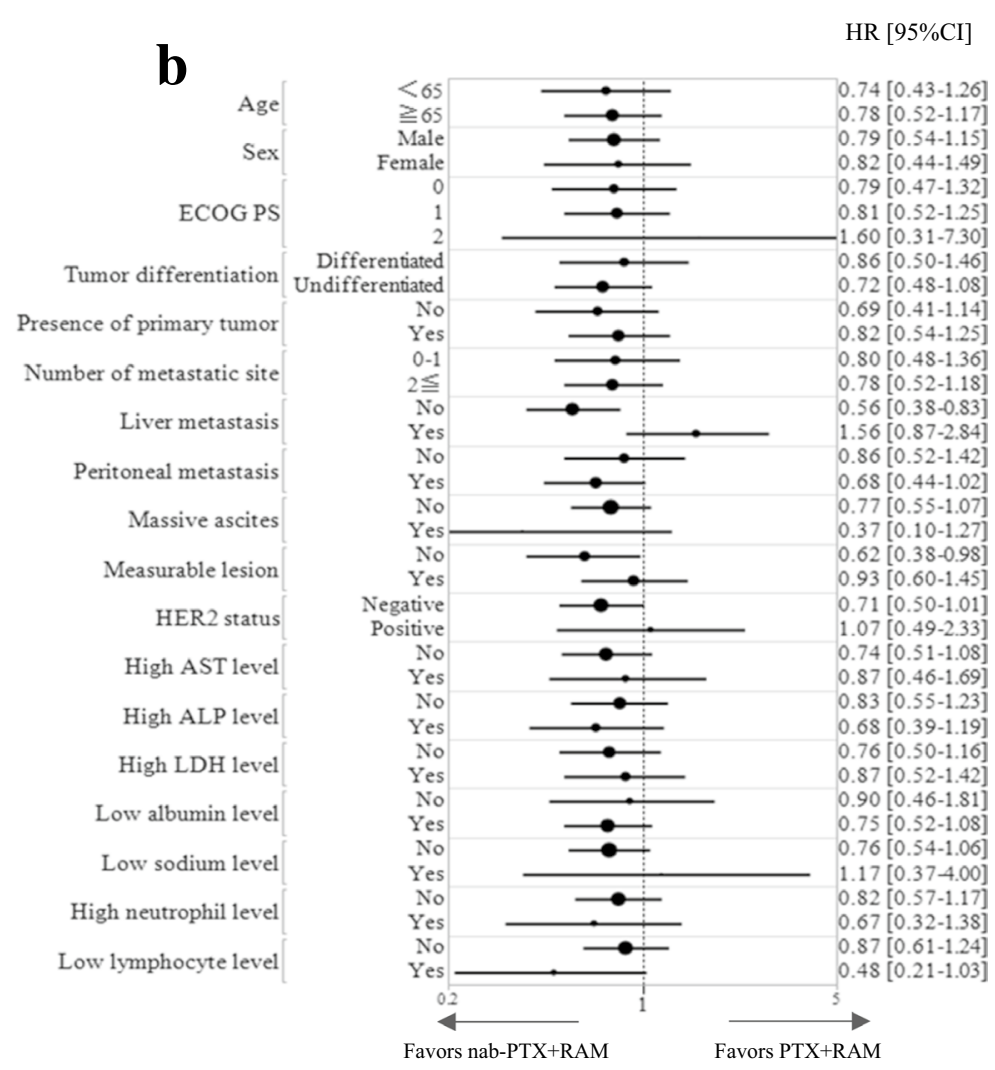

Interaction p-value

0.92

0.98

0.99

0.64

0.67

0.89

0.01

0.51

0.36

0.18

0.37

0.75

0.62

0.78

0.61

0.67

0.56

0.13 
Table 2 Tumor response

\begin{tabular}{lllll}
\hline $\begin{array}{llll}\text { nab- } & \text { PTX+RAM } & \text { Fisher's exact test } \\
\quad \% & N=48 \quad \% & p \text { value }\end{array}$ \\
\hline
\end{tabular}

Best overall respose

Complete response

Partial response

Stable disease

Progressive disease

Not evaluated

Overall response rate

Disease control rate

$\begin{array}{llll}0 & 0 & 1 & 2 \\ 21 & 40 & 17 & 35 \\ 25 & 47 & 19 & 40 \\ 6 & 11 & 11 & 23 \\ 1 & 2 & 0 & 0 \\ 21 & 40 & 18 & 37 \\ 46 & 87 & 37 & 77\end{array}$

$N a b-P T X+R A M$ nanoparticle albumin-bound paclitaxel plus ramucirumab, $P T X+R A M$ paclitaxel plus ramucirumab, $N$ number of patients

and trifluridine/tipiracil, differed between the two treatment groups. We speculated the main reason for the difference was the approval of nivolumab and trifluridine/tipiracil during the study period. They were approved as treatment for
AGC in Japan in September 2017 and August 2019, respectively. As nab-PTX + RAM has been currently selected more frequently, the patients administered nab-PTX + RAM were considered to have more chance to access these drugs.

The present study has several limitations. First, this was a non-randomized retrospective study, and the sample size was small. Although we used propensity score matching to balance the patient background characteristics in the treatment groups, we could not adjust for unmeasured confounding factors, which might have affected the results. In addition, the sample size shrank further when matched pairs were made, which weakened the statistical power. Second, as mentioned above, the difference in proportion receiving post-study treatment might have partly affected OS. Considering this bias, OS data should be interpreted with caution.

In conclusion, nab-PTX + RAM exhibited favorable trends in terms of PFS and OS compared with PTX + RAM. Although hematological toxicity was of concern, it was manageable. Based on these results, further studies including randomized-controlled studies are warranted.
Table 3 Adverse events $\geq$ Grade 3

\begin{tabular}{|c|c|c|c|c|c|}
\hline & \multicolumn{2}{|c|}{ nab-PTX + RAM } & \multicolumn{2}{|c|}{ PTX + RAM } & \multirow{2}{*}{$\begin{array}{l}\text { Fisher's exact test } \\
p \text { value }\end{array}$} \\
\hline & $N=95$ & $\%$ & $N=95$ & $\%$ & \\
\hline Any adverse events & 82 & 86 & 66 & 69 & $<0.01$ \\
\hline \multicolumn{6}{|l|}{ Hematological } \\
\hline Neutrophil count decreased & 68 & 72 & 53 & 56 & 0.03 \\
\hline White blood cell decreased & 30 & 32 & 30 & 32 & 1.00 \\
\hline Anemia & 25 & 26 & 16 & 17 & 0.15 \\
\hline Platelet count decreased & 5 & 5 & 4 & 4 & 1.00 \\
\hline \multicolumn{6}{|l|}{ Non-hematological } \\
\hline Febrile neutropenia & 8 & 8 & 9 & 9 & 1.00 \\
\hline Peripheral sensory neuropathy & 4 & 4 & 9 & 9 & 0.24 \\
\hline Fatigue & 4 & 4 & 3 & 3 & 1.00 \\
\hline Anorexia & 3 & 3 & 2 & 2 & 1.00 \\
\hline Mucositis oral & 1 & 1 & 1 & 1 & 1.00 \\
\hline Diarrhea & 1 & 1 & 1 & 1 & 1.00 \\
\hline Edema limbs & 1 & 1 & 2 & 2 & 1.00 \\
\hline Nausea & 0 & 0 & 1 & 1 & 1.00 \\
\hline Vomiting & 0 & 0 & 1 & 1 & 1.00 \\
\hline Bone infection & 1 & 1 & 0 & 0 & 1.00 \\
\hline Pneumonitis & 0 & 0 & 1 & 1 & 1.00 \\
\hline \multicolumn{6}{|l|}{ Special interest } \\
\hline Hypertension & 11 & 12 & 7 & 7 & 0.45 \\
\hline Proteinuria & 4 & 4 & 5 & 5 & 1.00 \\
\hline Gastrointestinal hemorrhage & 4 & 4 & 0 & 0 & 0.12 \\
\hline Thromboembolic event & 2 & 2 & 2 & 2 & 1.00 \\
\hline Gastrointestinal perforation & 0 & 0 & 2 & 2 & 0.49 \\
\hline
\end{tabular}

$N a b-P T X+R A M$ nanoparticle albumin-bound paclitaxel plus ramucirumab, $P T X+R A M$ paclitaxel plus ramucirumab, $N$ number of patient 
Supplementary Information The online version contains supplementary material available at https://doi.org/10.1007/s10147-022-02114-y.

Acknowledgements The authors would like to thank Editage (www. editage.jp) for the English language review.

Author contributions All the authors contributed to the study conception and design. Material preparation was performed by AN, TM, TK, TM, TN, NY, and IH. Data collection was performed by AN, YH, TI, YK, TM, YY, and TT. Analysis was performed by AN, NY, and IH. The first draft of the manuscript was written by AN, and all the authors commented on previous versions of the manuscript. All the authors have read and approved the final manuscript.

Data transparency The datasets analyzed during the current study are available from the corresponding author on reasonable request.

\section{Declarations}

Conflict of interest Akio Nakasya has no conflict of interest. Takeshi Kajiwara received honoraria for lectures from Chugai Pharmaceutical, Taiho Pharmaceutical, Bristol-Myers Squibb, Merck Biopharmaceutical, and Ono Pharmaceutical. Toshikazu Moriwaki received honoraria for lectures from Taiho Pharmaceutical, Bristol-Myers Squibb, and Eli Lilly. Tomohiro Nishina received honoraria for lectures from Chugai Pharmaceutical, Taiho Pharmaceutical, Ono Pharmaceutical, Takeda Pharmaceutical, and Daiichi-Sankyo Pharmaceutical. Ichinosuke Hyodo received payment for consulting services from Asahi-Kasei Pharmaceutical and honoraria for lectures from Chugai Pharmaceutical, Takeda Pharmaceutical, and Yakult-Honsha, and payment as a Safety Monitoring board member from Chugai Pharmaceutical, Taiho Pharmaceutical, Ono Pharmaceutical, Daiichi-Sankyo Pharmaceutical, and Merck Serano Pharmaceutical. Yuya Hagiwara, Tatsuki Ikoma, Yusuke Kurioka, Toshihiko Matsumoto, Yoshiyuki Yamamoto, Takao Tsuduki, and Natsumi Yamashita have no conflict of interest.

Ethics approval The study protocol was approved by the ethics committees of all participating institutions (approval number: 2020-65 at Shikoku Cancer Center, approval number: R03-022 at University of Tsukuba, approval number: 2020-46 at Himeji Red Cross Hospital, approval number: zn210121 at Kobe City Medical Center General Hospital). The requirement for informed consent was waived because the study design was retrospective. Opt-out opportunities were provided on each institution's website. This research was conducted in accordance with the tenets of the Declaration of Helsinki.

Open Access This article is licensed under a Creative Commons Attribution 4.0 International License, which permits use, sharing, adaptation, distribution and reproduction in any medium or format, as long as you give appropriate credit to the original author(s) and the source, provide a link to the Creative Commons licence, and indicate if changes were made. The images or other third party material in this article are included in the article's Creative Commons licence, unless indicated otherwise in a credit line to the material. If material is not included in the article's Creative Commons licence and your intended use is not permitted by statutory regulation or exceeds the permitted use, you will need to obtain permission directly from the copyright holder. To view a copy of this licence, visit http://creativecommons.org/licenses/by/4.0/.

\section{References}

1. Association JGC, Japanese gastric cancer treatment guidelines (2018) 5th ed. Gastric Cancer 2021(24):1-21

2. National Comprehensive Cancer Network (2020) NCCN clinical practice guidelines in oncology gastric cancer version 1

3. Smyth EC, Verheij M, Allum W et al (2016) Gastric cancer: ESMO clinical practice guidelines for diagnosis, treatment, and follow-up. Ann Oncol 27:v38-49

4. Wilke H, Muro K, Van Cutsem E et al (2014) Ramucirumab plus paclitaxel versus placebo plus paclitaxel in patients with previously treated advanced gastric cancer or gastro-oesophageal junction adenocarcinoma (RAINBOW): a double-blind, randomized phase 3 trial. Lancet Oncol 15:1224-1235

5. Shitara K, Takashima A, Fujitani K et al (2017) Nab-paclitaxel versus solvent-based paclitaxel in patients with previously treated advanced gastric cancer (ABSOLUTE): an open-label, randomized, non-inferiority, phase 3 trial. Lancet Gastroenterol Hepatol 2:277-287

6. Bando H, Shimodaira H, Fujitani K et al (2018) A phase II study of nab-paclitaxel in combination with ramucirumab in patients with previously treated advanced gastric cancer. Eur J Cancer 91:86-91

7. Ishikawa M, Iwasa S, Nagashima K et al (2020) Retrospective comparison of nab-paclitaxel plus ramucirumab and paclitaxel plus ramucirumab as second-line treatment for advanced gastric cancer focusing on peritoneal metastasis. Investig New Drugs 38:533-540

8. Okunaka M, Kotani D, Demachi K et al (2020) Retrospective cohort study of nanoparticle albumin-bound paclitaxel plus ramucirumab versus paclitaxel plus ramucirumab as second-line treatment in patients with advanced gastric cancer. BMC Cancer 20:1111

9. Eisenhauer EA, Therasse P, Bogaerts J et al (2009) New response evaluation criteria in solid tumours: Revised RECIST guideline (version 1.1). Eur J Cancer 45:228-247

10. Common Terminology Criteria for Adverse Events (CTCAE v5.0). Available at: http://www.evsncinih.gov/ftp1/CTCAE/ CTCAE_403_2010-06-14_QuickReference_5x7pdf. Accessed 27 Nov 2017

11. Chau I, Norman AR, Cunningham D et al (2004) Multivariate prognostic factor analysis in locally advanced and metastatic esophago-gastric cancer-pooled analysis from three multicenter, randomized, controlled trials using individual patient data. J Clin Oncol 22(12):2395-2403

12. Takahari D, Boku N, Mizusawa J et al (2014) Determination of prognostic factors in Japanese patients with advanced gastric cancer using the data from a randomized controlled trial, Japan clinical oncology group 9912. Oncologist 19(4):358-366

13. Fuchs CS, Muro K, Tomasek J et al (2017) Prognostic factor analysis of overall survival in gastric cancer from two Phase III studies of second-line ramucirumab (REGARD and RAINBOW) using pooled patient data. J Gastric Cancer 17:132-144

14. Austin PC (2009) Balance diagnostics for comparing the distribution of baseline covariates between treatment groups in propensity-score matched samples. Stat Med 28(25):3083-3107

15. Takashima A, Shitara K, Fujitani K et al (2019) Peritoneal metastasis as a predictive factor for nab-paclitaxel in patients with pretreated advanced gastric cancer: an exploratory analysis of the phase III ABSOLUTE trial. Gastric Cancer 22:155-163

16. Hirata K, Hamamoto Y, Ando M et al (2020) Weekly paclitaxel plus ramucirumab versus weekly nab-paclitaxel plus ramucirumab 
for unresectable advanced or recurrent gastric cancer with peritoneal dissemination refractory to first-line therapy-the P-select trial (WJOG10617G) - a randomized phase II trial by the West Japan Oncology Group. BMC Cancer 20(1):548

17. Shitara K, Matsuo K, Takahari D et al (2010) Neutropenia as a prognostic factor in advanced gastric cancer patients undergoing second-line chemotherapy with weekly paclitaxel. Ann Oncol 21:2403-2409
18. Shitara K, Yuki S, Takahari D et al (2014) Randomised phase II study comparing dose-escalated weekly paclitaxel vs standard-dose weekly paclitaxel for patients with previously treated advanced gastric cancer. Br J Cancer 110:271-277

Publisher's Note Springer Nature remains neutral with regard to jurisdictional claims in published maps and institutional affiliations. 